\title{
O USO OFF LABEL DE MEDICAMENTOS PARA O TRATAMENTO DA OBESIDADE NO BRASIL
}

\section{OFF LABEL USE OF DRUGS FOR THE TREATMENT OF OBESITY IN BRAZIL}

\author{
Larissa Souza dos Reis Gonçalves ${ }^{1}$ \\ Thiago Pereira de Abreu²
}

RESUMO: A obesidade é uma doença crônica que é caracterizada pelo acúmulo excessivo de gordura no corpo. Uma pessoa é considerada obesa quando seu índice de massa corporal é igual ou maior a $30 \mathrm{~kg} / \mathrm{m}^{2}$. O excesso de peso está presente em $60 \%$ dos brasileiros e segue em elevação. Atualmente as opções de medicamentos para o tratamento da obesidade são escassas e não se adaptam bem a todos os indivíduos. Por conta disso, a prescrição de medicamentos off label surge como alternativa, devendo ser realizada de forma individualizada a cada paciente. $O$ artigo busca descrever e analisar o uso dos principais medicamentos off label mais prescritos para a obesidade no Brasil, discutindo os aspectos relacionados a necessidade do seu uso através da metodologia de revisão de artigos.

Palavras -Chave: Obesidade. Tratamento. Medicamentos. Off label.

ABSTRACT: Obesity is a chronic disease, which is characterized by excessive fat accumulation in the body. A person is considered obese when their body mass index is equal to or greater than $30 \mathrm{~kg} / \mathrm{m}^{2}$. Overweight is present in $60 \%$ of Brazilians. Nowadays, the options of medications for obesity treatment are scarce and do not adapt well to all individuals, because of this, the lack of off label medications appears as an alternative, and should be carried out individually for each patient. The article seeks to describe and analyze the use of the main off label drugs most prescribed for obesity in Brazil, discussing the aspects related to the need of their use through the article review methodology.

Keywords: Obesity. Treatment. Medications. Off label.

\footnotetext{
${ }^{1}$ Universidade Iguaçu (UNIG) -Graduação em Farmácia.

${ }^{2}$ Universidade Federal do Rio de Janeiro (UFRJ) -Graduação em Farmácia. Instituto de Biofísica Carlos ChagasFilho (IBCCF -UFRJ) -Pós-graduação em Fisiologia. School of Medicine na Johns Hopkins University (Baltimore EUA) -Doutorado em Fisiologia.
} 


\section{INTRODUÇÃO}

A obesidade é uma doença crônica, definida pela Organização Mundial de Saúde (OMS) como o acúmulo anormal ou excessivo de gordura no corpo. Uma pessoa é considerada obesa quando seu Índice de Massa Corporal (IMC) é maior ou igual a 30 $\mathrm{kg} / \mathrm{m}^{2}$ e a faixa de peso normal varia entre 18,5 e $24,9 \mathrm{~kg} / \mathrm{m}^{2}$. Só no Brasil, cerca de $20 \% \mathrm{da}$ população tem obesidade. $\mathrm{O}$ excesso de peso está presente em $60 \%$ dos brasileiros adultos, segundo o IBGE e essas estatísticas estão em elevação em todas as faixas etárias. (BVS MINISTÉRIO DA SAÚDE, 2020)

A Organização Mundial da Saúde (OMS) estima que cerca de 4 milhões de pessoas morrem todos os anos em decorrência de doenças causadas pelo excesso de peso. Além disso, a obesidade é um agravante para outras doenças como as cardiovasculares, diabetes e até mesmo alguns tipos de câncer. (CURY, R7, 202I)

Imagem r: O conceito de Obesidade está relacionado ao IMC (índice de massa corporal), esse cálculo é feito dividindo-se o peso em $\mathrm{kg}$ pela altura em metros, elevada ao quadrado. IMCs acima de 30 já são considerados como obesidade.
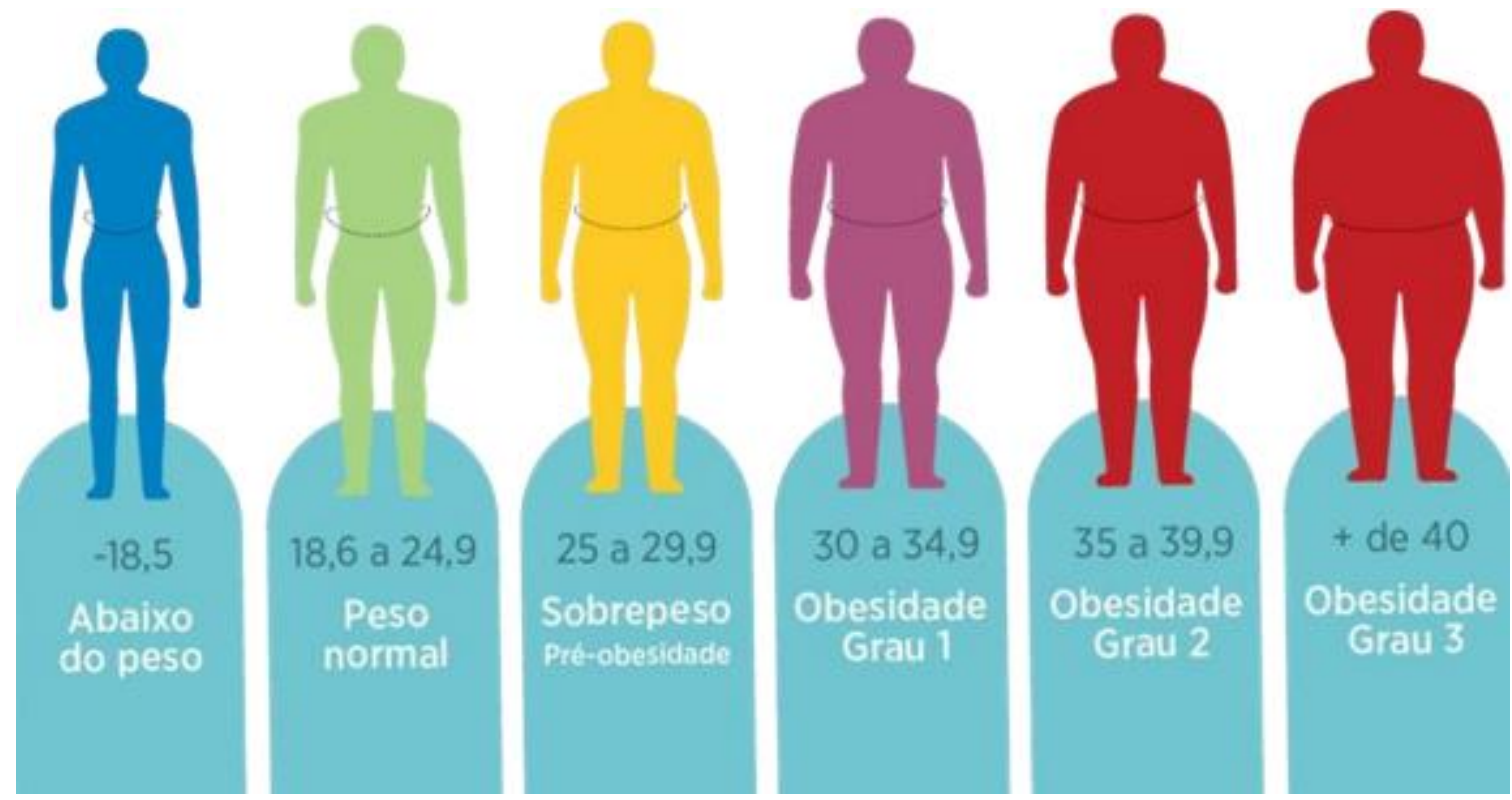

Fonte: portal CBN Campinas 
Entre as causas da obesidade, podemos apontar multifatores, sendo eles biológicos, econômicos, ambientais, sociais, culturais e políticos, sobre as quais o indivíduo possui pouca ou nenhuma capacidade de interferência. Também está relacionada à má alimentação, ao sedentarismo e ao modo de viver na atualidade. A base do tratamento são mudanças no estilo de vida, como dieta e exercícios, podendo-se associar o uso de medicamentos. No entanto, o percentual de pacientes que não obtêm resultados satisfatórios com medidas conservadoras é alto. (ABESO, 2016)

Atualmente, no Brasil, há poucos medicamentos registrados para o tratamento da obesidade, são eles: Liraglutida, Lorcasserina, Sibutramina e Orlistate. Os medicamentos atuam no controle do apetite e alguns até buscam reduzir a absorção de gordura pelo organismo. Porém, alguns outros medicamentos, liberados para outras finalidades estão sendo amplamente prescritos.

No universo farmacêutico, estudamos de forma abrangente as ciências da natureza; a Lei de Lavoisier indica que "na natureza nada se cria nem se copia, tudo se transforma". Tendo isso em mente, seria errado pensar que a criação de uma droga-um composto químico complexo - seria restrita a apenas uma função, sendo que ela pode ser adaptada para outra finalidade, depois de estudos e revisões. Os medicamentos que são indicados para finalidades diferentes daquelas aos quais estão descritas na bula ou que não são regulamentados são o que chamamos de off label, ou em tradução literal 'fora da bula". Com isso, quando um medicamento é aprovado pela Anvisa como indicado para determinada função isso não significa que seja a única possível, podendo ser posteriormente adaptado para faixas etárias diferentes, para outras doenças e até mesmo ter o seu uso restrito. Seu uso não é proibido, porém é realizado por conta e risco do médico prescritor, não sendo caracterizado - na grande parte das vezes - como erro médico, podendo possuir base em evidências de estudos que não foram regulamentados ainda, tendo seu uso adaptado e individualizado a cada paciente. (ANVISA, 2021) 


\section{OBJETIVO}

\subsection{Objetivo geral}

Sendo a obesidade um problema de saúde crescente no Brasil, este artigo tem como objetivo descrever e analisar os principais medicamentos off label prescritos para o tratamento da obesidade, discutindo os aspectos que levam a necessidade do seu uso.

\subsection{Objetivos específicos}

- Averiguar a importância do uso off labels de medicamentos no tratamento da obesidade;

- Identificar a necessidade do seu uso no tratamento individualizado ao paciente;

- Quando utilizar e quando não utilizar o tratamento off label;

- Atuação do farmacêutico no acompanhamento do paciente com o tratamento off label.

\section{JUSTIFICATIVA}

A Reflexão a cerca do uso de medicamentos off label no tratamento da obesidade possui interesses das mais diversas partes envolvidas nesse processo, desde o órgão regulatório, médico, paciente e a área farmacêutica. É importante ressaltar os eventuais riscos e benefícios desta prática, bem como a necessidade do seu uso.

Com o objetivo de atrair atenção para o tema, o trabalho busca apontar a melhor maneira de lidar com essa prática, de forma com que a segurança e bem estar do usuário seja o maior objetivo.

\section{O USO DE MEDICAMENTOS OFF LABEL PARA A OBESIDADE NO BRASIL}

O registro de novos medicamentos é concedido após a comprovação da segurança, eficácia e qualidade do mesmo, sendo que as duas primeiras devem ser baseadas na avaliação de estudos clínicos e testes que comprovem essas indicações. A aprovação de novos medicamentos no Brasil, porém, pode demorar, por vários motivos, entre os quais o 
de que o pedido de registro pode ser feito muito mais tarde aqui do que em outros países. Também pode ocorrer que o medicamento receba aprovação acelerada em outro país, baseada na apresentação de estudos preliminares ou incompletos, o que, via de regra, não é aceito pela Anvisa. Por fim, um uso autorizado do medicamento no Brasil pode ser uso off label em outros países. (ANVISA, 202I)

\section{I Principais medicamentos off labels utilizados no tratamento da obesidade no Brasil}

O tratamento farmacológico da obesidade só deve ser feito mediante orientação médica e deve ser realizado de forma individualizada a cada paciente, com uma dieta específica e mudanças no estilo de vida. Essa associação ajuda a aumentar a adesão dos pacientes ao tratamento e ter resultados significativos. Deve-se ter em mente que o medicamento não cura a obesidade, e sim busca auxiliar na perca do peso excessivo. $O$ tratamento deve ser mantido apenas quando considerado seguro e efetivo para o paciente em questão. (Mancini; Halpern, 2002)

Atualmente as opções de medicamentos aprovados para o tratamento da obesidade não se adaptam bem a todos os indivíduos. Dentre os medicamentos on-label - que são aprovados e regulamentados pela Anvisa -o mais conhecido para o tratamento da obesidade é a Sibutramina. Criada inicialmente como antidepressivo, ela age no sistema nervoso central, especialmente sobre dois neurotransmissores, a serotonina e a noradrenalina. Ela provoca no paciente a sensação de saciedade e o controle da fome. $\mathrm{O}$ risco que o uso indiscriminado provoca no organismo é muito alto, já que a administração do medicamento em pessoas com problemas cardíacos pode levar ao derrame e infarto, além de uma infinidade de efeitos colaterais, como a boca seca e amarga, náusea, estômago irritado, constipação, problemas para dormir, tontura, dores menstruais, dor de cabeça, sonolência, alteração de humor e dor nos músculos e nas articulações.(Gr,2013) 
Imagem 2: A associação da terapia medicamentosa aliada a mudanças no estilo de vida tem se mostrado eficaz na perca de peso excessivo. A prescrição off label pelo médico surge como alternativa para o tratamento $\mathrm{da}$ obesidade quando os medicamentos regulamentados não se adaptam ou são contraindicados para determinados pacientes.

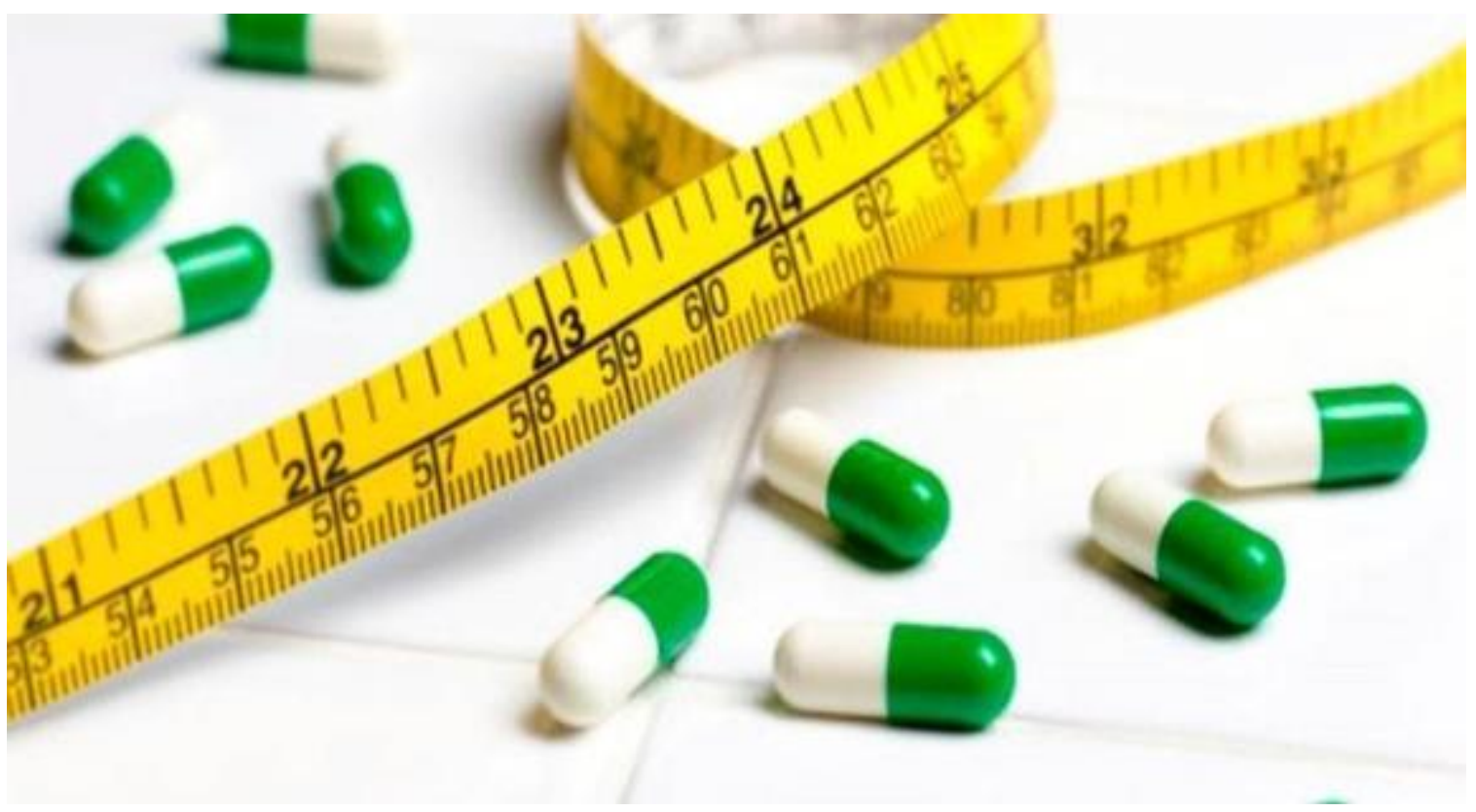

Fonte: Revista Saúde

Entre os principais fármacos prescritos, encontram-se anticonvulsivantes, como o topiramato, fármacos utilizados no controle de diabetes, como metformina e antidepressivos, como fluoxetina e bupropiona. (Zaros,2018).

\subsection{Topiramato}

O topiramato é um medicamento aprovado para uso como agente antiepiléptico e que também se mostra útil para o controle das crises de enxaqueca, porém, foi observado que após o uso do fármaco, os pacientes apresentaram perda de peso, principalmente por aqueles que tinham compulsão alimentar. Após pesquisas realizadas e vários ensaios clínicos comprovaram que realmente causava perda de peso nos indivíduos. Sua indicação off label é indicada para aqueles indivíduos que possuem o "comer emocional".

Acredita-se que o seu mecanismo de ação no efeito emagrecedor tenha ação no aumento do ácido gama-aminobutírico (GABA), principal neurotransmissor inibidor do 
sistema nervoso central. Com isso, inibindo a anidrase carbônica, aumentaria a catecolamina disponível (noradrenalina e dopamina), que age nos núcleos laterais do hipotálamo, isso resultaria na redução de apetite e como consequência, a diminuição no consumo de alimentos.

Suas principais contraindicações são para indivíduos que possuam histórico de litíase renal, glaucoma de ângulo fechado e mulheres em período fértil que estejam tentando engravidar, por possuir risco de toxicidade fetal. (Giorelli, 2020)

\subsubsection{Topiramato associado a fentermina}

A associação do topiramato com fentermina - disponível com o nome comercial Qsymia - tem o seu uso aprovado pela FDA (food and drug administration), agência federal que regulamenta o serviço de saúde nos Estados Unidos, porém ainda não possui seu uso regulamentado no Brasil.

O objetivo dessa associação tem o intuito de prolongar o efeito dos fármacos no organismo, fazendo com que tenha a melhora da resposta terapêutica. (Giorelli, 2020)

\subsubsection{Bupropiona}

Indicado como antidepressivo, observou-se em trabalhos científicos que o uso desse fármaco favorecia a perda de peso dos indivíduos que já o utilizavam. Seu uso associado a naltrexona é aprovado pela FDA, com o nome comercial Contrave, porém ainda não é regulamentada no Brasil, possuindo assim, o seu uso off label.

Tem o seu mecanismo de ação inibindo a recaptação pré-sináptica da noradrenalina e dopamina. Com isso, se encontram mais disponível dentro da fenda sináptica, aumentando a sua captação pelo neurônio pós-sináptico. Esses dois nutroneurotransmissores agem no sistema nervoso central, aumentando a sensação de bem estar, o que inibiria a fome. (Giorelli, 2020). 


\subsubsection{Bupropiona associada a naltrexona}

A associação desses dois medicamentos se mostrou eficaz, pois o nível de proopiomelanocortina - neurotransmissor de neurônios de primeira ordem, que possui efeito anorexígeno - diminuía com o tempo. Isso ocorre pois a bupropiona forma uma beta-endorfina (opióide) que inibe o efeito anorexígeno desse neurotransmissor ao longo do tempo de uso. A naltrexona age como um bloqueador opioide, aumentando o efeito da mesma e favorecendo uma maior perca de peso. (Giorelli, 2020).

\subsubsection{Fluoxetina}

A Fluoxetina é um medicamento aprovado como sendo antidepressivo e ansiolítico. Age como inibidor seletivo da receptação de serotonina. Seu uso off label tem sido indicado para caso de indivíduos com compulsão alimentar, sendo o efeito emagrecedor um efeito colateral desse medicamento. Esse efeito é limitado a fase aguda inicial (3-6 meses), podendo causar efeito rebote posteriormente. (ABESO, 2016).

\subsubsection{Metformina}

O cloridrato de metformina é um medicamento antidiabético de uso oral, que associado a uma dieta apropriada, é utilizado para o tratamento do diabetes tipo 2. O uso da metformina pode auxiliar no emagrecimento pois controla a quantidade de glicose no sangue ao decorrer do tempo, o que ajuda a controlar a fome entre as refeições, podendo promover a perda de peso.

Um estudo realizado que buscava avaliar o uso da metformina na perda de peso em indivíduos não diabéticos com obesidade (C. Seifarth, B. Schehler, H. J. Schneider, 2013) concluiu que a droga se mostrou eficaz em um ambiente ambulatorial naturalista em pacientes obesos e resistentes a insulina.

Contudo, a metformina tem efeitos colaterais como náuseas, cansaço e vômitos e algumas contra indicações. $\mathrm{O}$ uso do componente também é contraindicado para pacientes que tenham insuficiência renal ou doença hepática grave. 


\section{PAPEL DO PROFISSIONAL FARMACÊUTICO NO TRATAMENTO OFF LABEL}

O farmacêutico é o profissional de saúde capacitado de orientar os pacientes sobre tudo o que abrange a área de medicamentos. É uma fonte confiável de conhecimento não apenas para pacientes, mas também para outros profissionais da área de saúde, garantindo que o medicamento correto seja fornecido ao paciente da forma mais adequada, tanto na sua dose quanto na sua formulação.

Um bom diálogo com esse profissional pode colaborar como motivação ao cumprimento do tratamento, otimizando a utilização da farmacoterapia. Também auxilia na orientação sobre os efeitos adversos que o medicamento possa causar, principalmente no caso do uso off label.

Imagem 3: $O$ farmacêutico ao avaliar o estado de saúde do paciente tem como incentivá-lo a aderir ao tratamento prescrito pelo seu médico com segurança.

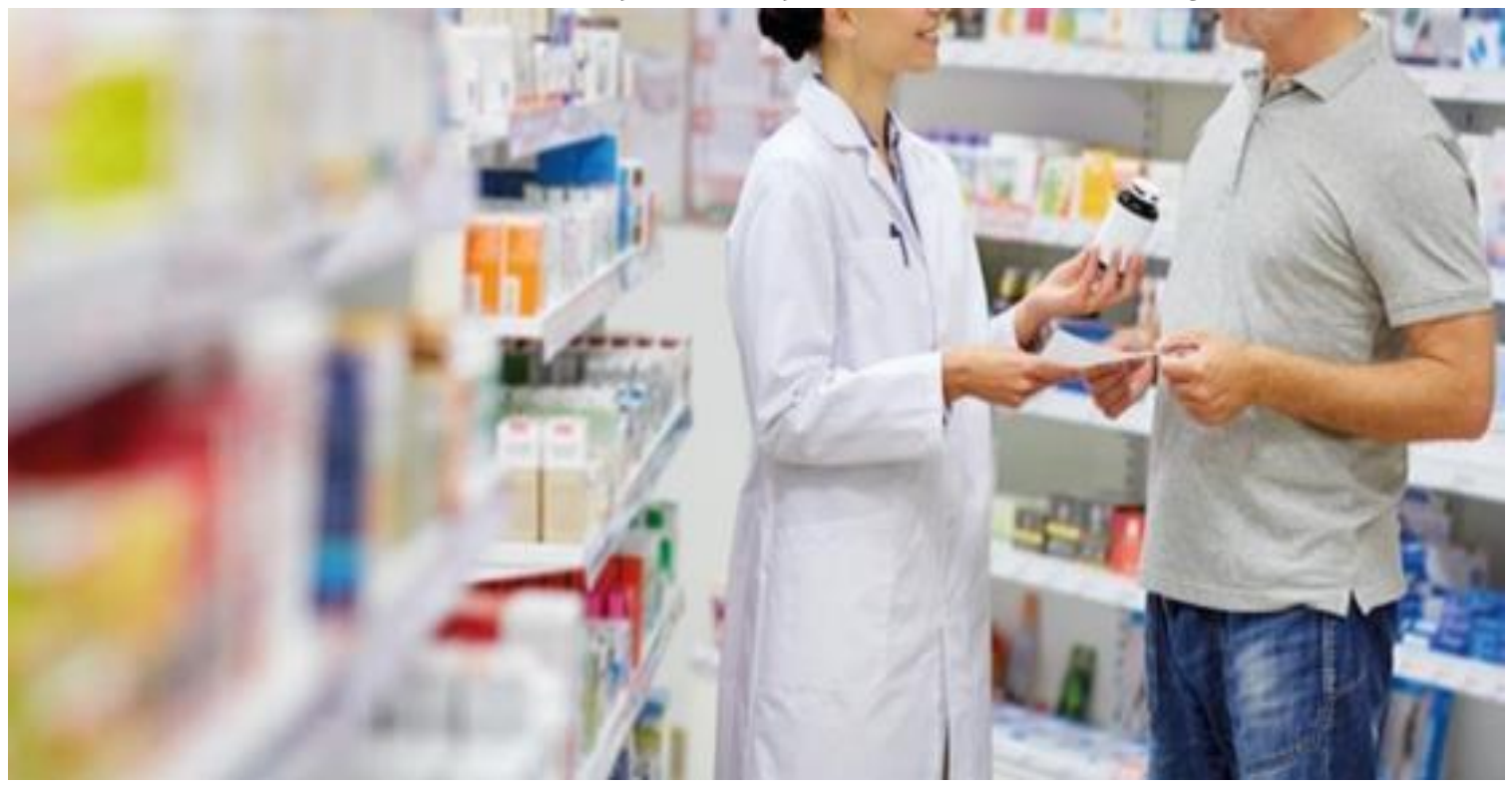

Fonte: Revista Saúde.

Seu objetivo é proporcionar cuidado e segurança ao paciente, promovendo o uso racional dos medicamentos, buscando alcançar resultados que melhorem a qualidade de vida do indivíduo. 


\section{METODOLOGIA}

Trata-se de um estudo de pesquisa exploratória, com abordagem de resultados qualitativa, pois possui como finalidade buscar descrever e analisar o uso de medicamentos off label, discutindo os aspectos relacionados a necessidade do seu uso. Foi realizada a revisão bibliográfica de artigos datados entre 2013-2021, utilizando a ferramenta de pesquisa Google Acadêmico e análise de documentos e revistas científicas.

\section{RESULTADOS}

Os medicamentos off label se apresentam como alternativa válida no auxílio ao tratamento da obesidade no Brasil quando houver evidência de benefício ao paciente e quando a terapia padrão for inadequada. Esses medicamentos são amplamente prescritos por endocrinologistas com base em experiências anteriores de pacientes. $O$ tratamento deve ser prescrito de forma individualizada, se o paciente se adequar as especificidades do uso do fármaco, devendo possuir supervisão médica ao decorrer do uso.

\section{DISCUSSÃO}

Ao longo do trabalho desenvolvido foi constatado que a prática off label pode vir a ser benéfica no tratamento da obesidade, para os pacientes que não se adequam ao tratamento convencional, sendo utilizado como de forma alternativa. Esse uso tem sido essencial para a evolução das pesquisas cientificas sobre fármacos.

A aprovação de um medicamento no Brasil passa por processo bastante burocrático, que abrange diversas etapas, necessárias, porém, torna a aprovação demorada o que não é muito interessante para as companhias farmacêuticas.

Todo medicamento utilizado deve ter como objetivo garantir ou preservar a saúde do paciente e como um medicamento pode ser aprovado em outro país e ter o seu uso regulamentado, seria errado impedir o uso do mesmo fármaco ou forma farmacêutica ao individuo que precisa se assim for possível. $O$ uso deve ser sempre baseado em evidências e com segurança ao indivíduo. 


\section{CONCLUSÕES}

A obesidade é um problema complexo e somente o uso da farmacoterapia como tratamento não soluciona o problema, que deve ser associado a acompanhamento nutricional, atividades físicas e em muitos casos, acompanhamento psicológico. O uso off label de medicamentos para a obesidade pode ser utilizado de forma alternativa, quando a terapêutica padrão não for recomendada ao indivíduo. Sendo assim, cada prescrição deve ser feita de forma individualizada, visando o bem estar do paciente. O paciente deve estar ciente de que aquele medicamento não é aprovado pela Anvisa para essa indicação. A atuação do farmacêutico em orientar o paciente é fundamental para promover o uso racional desses medicamentos.

\section{REFERÊNCIAS}

Biblioteca virtual em saúde, Ministério da Saúde. 04/o3, dia mundial da obesidade. Disponível em https://bvsms.saude.gov.br/o4-3-dia-mundial-da-obesidade/ Acesso em agosto de 2021

Cury, Ana Carolina. Pandemia contribuiu para o aumento do número de obesos no Brasil - portal r7, 2021 Disponível em: 〈https://noticias.r7.com/brasil/pandemiacontribuiu-parao-aumento-do-numero-de-obesos-no-brasil-23072021> Acesso em agosto de 2021

Gerência de medicamentos novos, pesquisa e ensaios clínicos. Como a anvisa vê o uso off label de medicamentos. Disponível em: <http://antigo.anvisa.gov.br/en_us/resultadodebusca?p_p_id=Ior\&p_p_lifecycle $=$ o\&p_p_state $=$ maximized $\&$ p_p_mode $=$ view $\&$ p_p_col_id $=$ columnI\&P_P_col_count $=$ I\&_IOI_struts_action $=\% 2$ fasset_publisher\%2fview_content $\&$ _IOI_ assetentryid $=352702 \&$ _IoI_type $=$ content $\&$ _IoI_groupid $=33836 \&$ _IoI_urltitle $=$ como-a-anvisa ve-o-uso-off-label-

demedicamentos\&redirect $=$ http\%3a\%2f\%2fantigo.anvisa.gov.br\%2fen_us\%2fresultadodebu sca\%3fp_p_id\%3d3\%26p_p_lifecycle\%3do\%26p_p_state\%3dnormal\%26p_p_mode\%3dview\%2 6p_p_col_id\%3dcolumni\%26p_p_col_count\%3d1\%26_3_advancedsearch\%3dfalse\%26_3_group id\%3do\%26_3_keywords\%3dsemi\%25c3\%25airio\%2bvolta\%2ba\%2bdiscutir\%26_3_assetcateg 
oryids\%3d34506\%26_3_delta\%3d20\%26_3_resetcur\%3dfalse\%26_3_cur\%3d6\%26_3_struts_acti on\%3d\%252fsearch\%252fsearch\%26_3_format\%3d\%26_3_andoperator\%3dtrue\%26_3_formdat e\%3di44I824476958\&inheritredirect=true $>$ Acesso emagosto de 202I

Associação brasileira para o estudo da obesidade e da síndrome metabólica. Diretrizes brasileiras de obesidade 2016. 4. ed. são paulo: abeso, 2016. Disponível em: $<$ https://abeso.org.br/wp-content/uploads/2019/12/diretrizes-downloaddiretrizesbrasileiras-de-obesidade-2016.pdf > Acesso em setembro de 202I

Zaros, karin juliana. O uso off label de medicamentos para obesidade - cim/crfpr,2018. Disponível em: 〈https://www.crfpr.org.br/uploads/revista/33657/cewoqhorzwusjg2f 4 iomlihrfogf2etv.pdf〉 Acesso em setembro de 202I

MANCINI, Marcio C. ; HALPERN, Alfredo. Tratamento Farmacológico da Obesidade. USP, São Paulo, 2002. Disponível em: https://www.scielo.br/j/abem/a/HZjBXh5JRcHdSBcXxYMrNVp/?lang=ptAcesso em setembro de 2020

BEZERRA, Camila. A eficácia do tratamento com topiramato em pacientes com obesidade. $\mathrm{HGF}$ 202I. Disponível em: 〈http://extranet.hgf.ce.gov.br/jspui/bitstream/r23456789/521/I/CAMILA\%2oMONTEIR O\%20TCR\%2oENDOCRINOLOGIA.pdf> Acesso em setembro 202I

Giorelli, Paulo. O uso do topiramato em obesidade. Dr. Giorelli - Youtube, 29 de junho de 2020. Disponível em 〈https://www.youtube.com/watch?v ${ }_{7} \mathrm{PNf}_{3} \mathrm{X6} 6_{1}$ bo $>$ Acesso em setembro de 202I

C. Seifarth, B. Schehler, H. J. Schneider. Effectiveness of Metformin on Weight Loss in Non-Diabetic Individuals with Obesity. Experimental and Clinical Endocrinology \& Diabetes. Vol.121. I.ed; 27-31, 2013. Disponível em: 〈https://www.thiemeconnect.com/products/ejournals/html/Io.1055/s-0032-1327734> Acesso em setembro de 2021 
PEREIRA; FREITAS; A evolução da Atenção Farmacêutica e a perspectiva para o Brasil. Rev. Bras. Cienc. Farm. 44 (4), Dez 2008. Disponível em: < https://doi.org/10.159o/Si51693322008000400006> Acesso em setembro de 202I

O papel social e profissional do farmacêutico como um especialista no cuidado à saúde. Centro de oncologia do abc. Disponível em: 〈https://oncologiaabc.com.br/importanciafarmaceutico/> Acesso em setembro de 2021 